\title{
Factors that Affects Male Partner Involvement in PMTCT Services in Africa: A Review Literature
}

\author{
Addis Adera Gebru ${ }^{1, ~ *}$, Mesfin Wudu Kassaw ${ }^{1}$, Yonas Yimam Ayene ${ }^{1}$, \\ Zemenu Mengistie Semene ${ }^{1}$, Markos Kidane Assefa ${ }^{2}$, Ambachew Woreta Hailu ${ }^{3}$ \\ ${ }^{1}$ Department of Nursing, Faculty of Health Sciences, Woldia University, North Wollo, Ethiopia \\ ${ }^{2}$ Huwawei Company, Addis Ababa Woreda Net project, Addis Ababa, Ethiopia \\ ${ }^{3}$ Department of Biology, Faculty of Natural and Computational Sciences, Woldia University, North Wollo, Ethiopia
}

\section{Email address:}

addisaderagebru@gmail.com (A. G. Addis), mesfine12a@gmail.com (W. K. Mesfin), yonasyimam@gmail.com (Y. A. Yonas), markos.kidane@yahoo.com (K. A. Markos), zemenumengistie@yahoo.com (M. S. Zemenu), ambalake@gmail.com (W. H. Ambachew)

\section{To cite this article:}

Addis Adera Gebru, Mesfin Wudu Kassaw, Yonas Yimam Ayene, Zemenu Mengistie Semene, Markos Kidane Assefa, Ambachew Woreta Hailu. Factors that Affects Male Partner Involvement in PMTCT Services in Africa: A Review Literature. Science Journal of Public Health. Vol. 3, No. 4, 2015, pp. 460-467. doi: 10.11648/j.sjph.20150304.13

\begin{abstract}
Back ground: male partner's participation in prevention of mother-to-child-transmission (PMTCT) of HIV has been determined as one of the major factors in world. And it realization is challenges because of male related and institutional factors. Objectives: This paper summarizes the factors which affects male partner's involvement in PMTCT services in Africa. Methods: A narrative Literature research was carried out of for evaluation of the literature generated from EBESCO systems, PUBMED, OVID, CINAHL, MEDLINE and GOOGLE SEARCH, and the Internet from January, 2010 to April, 2015. The literature reviewed suggests that male partner's participation in PMTCT of HIV and associated factors. The inclusion criteria were an original study or review studies involving male partner's participation in PMTCT of HIV and associated factors. Among selected papers were screened and irrelevant studies were excluded. Result: We included 19 studies in this review, which reported on factors which affects male partner's involvement in PMTCT services in Africa. The majority of studies described male partner participation is a crucial component to optimize ANC/PMTCT services. Other studies defined that the importance of male involvement in PMTCT is accepted. Conclusion: Factors which affects male partner's involvement in PMTCT services is a well-accepted issue and has important positive outcomes several areas of health discipline. The current literature, research and reviewed articles which were developed through an evaluation of this literature reviewed articles and the assessment of a limited number of research studies that focused on male partner's participation in PMTCT of HIV and associated factors in PMTCT services at different health settings. Implication of PMTCT services: It is proposed that male partner's participation in PMTCT of HIV improves as the PMTCT services gains HIV testing experience in their facilities with couples, male partner's gain a sense of saliency in relation to PMTCT services. Male partners may use PMTCT services independently, and concurrently to solve weak and longtime PMTCT services at different health Institutes.
\end{abstract}

Keywords: Male, Male Involvement, PMTCT, HIV, ANC, Associated Factors

\section{Introduction}

Male participation in the prevention of mother-to-child transmission (PMTCT) of HIV has been determined as one of the key factors in sub-Saharan African countries, but it realization is challenging because of male related and institutional factors (1).In 2009, of 125 million pregnant women in low and middle income countries, an estimated 1.4 million were living with human immunodeficiency virus (HIV) infection (2). Ethiopia is one of the top 20 countries affected by HIV in the World. Not only a proportion of women receiving antiretroviral prophylaxis in PMTCT program are small, but also adherence among the users is poor. The utilization and adherence of PMTCT services by the pregnant women may be influenced both by factors related to health system and individual factors (3).Despite the availability of a dual therapy treatment protocol and infant feeding guidelines designed to prevent mother-to-child transmission(PMTCT) of HIV, of the over 1 million babies born in South Africa each year ,only $70 \%$ of those born to 
HIV positive mothers received dual therapy(4).The intervention to prevent mother-to-child transmission(PMTCT) of HIV are effective in ensuring a healthy child and keeping mothers alive, there are many challenges to achieving successful interventions in Cameroon(5). Antenatal care(ANC) is a major entry point for PMTCT programs especially in countries with a high prevalence of HIV . The World Health Organization(WHO) recommends four ANC visits during pregnancy, which allows for early diagnosis, prevention or treatment of conditions which may jeopardize the health of the mother or her unborn baby(6,7).Male partner participation is a crucial component to optimize antenatal care/prevention of mother-to-child-transmission/PMTCT services. It creates an opportunity to capture pregnant mothers and their male partners to reverse the transmission of HIV during pregnancy, labour, and breast feeding (8).Mother-to-child transmission of HIV (PMTCT) accounts for over $95 \%$ of all pediatric HIV infections worldwide. Several studies have shown that male participation in the antenatal care of their spouses together with -couple counseling and testing for HIV, increase use of the interventions for HIV prevention (9).Male involvement in the PMTCT of HIV services is essential in a patriarchal society where men are decision makers of the household (10).Prevention of mother-to-child transmission of HIV epidemic. Male involvement in PMTCT influences the uptake of the program by their partners. However, male involvement in PMTCT programs has been characterized by low male involvement, prisons custodial staff being of no exception. There is no data to show factors, associated with limited male involvement in PMTCT by custodial staffs (11).

\section{Methods}

A narrative Literature research was carried out of for evaluation of the literature generated from EBESCO systems, PUBMED, OVID, CINAHL, MEDLINE and GOOGLE SEARCH, and the Internet from January, 2010 to April, 2015.The literature reviewed suggests that male partner's participation in PMTCT of HIV and associated factors. The inclusion criteria were an original study or review studies involving male partner's participation in PMTCT of HIV and associated factors. Among selected papers were screened and irrelevant studies were excluded.

\section{The PRISMA Diagram in Figure 1. Summarizes the Selection Process at Each Stage}

27 studies were retained for data extraction ,of which 19 reported data needed in this qualitative report(See Fig.1) .There was some disagreement between the two authors on studies to be included(Kappa estimate $0.57,95 \% \mathrm{CI}, \quad 0.41-0.74 ; \mathrm{P}<0.001)$. This agreement was resolved by discussion. The Kappa estimate for agreement on the methodological quality of included studies was $0.84,95 \%$

\section{CI, 0.74-0.94; $\mathrm{P}<0.001$}

This reviewed study were designed regarding the sequential outline of the review: (1)Prevalence and implementation of PMTCT, (2) Level of male involvement in PMTCT, Importance, utilization and adherence of PMTCT services,(3) Knowekldge about PMTCT and reason that rationalize male partner involvement in ANC/PMTCT, (4) Determinants of male involvement and association between male partner involvement in PMTCT and the uptake of PMTCT intervention,(5) Husbands accompany their wives to the ANC/PMTCT clinic, and (6) Programmatic factor or male friendly of the health facility ,husbands accompany their wives to the ANC/PMTCT clinic and Strategies of PMTCT

\subsection{Prevalence and Implementation of PMTCT}

The early infant diagnosis (EID) programs indicate an HIV prevalence of $8.8 \%$ among infants born to HIV positive mothers (12) Acknowledging a high HIV prevalence (8.0\%) among pregnant women in Cameroon, and the potential risks of PMTCT, an increase in MCH services use in health facilities with PMTCT services will improve maternal and child health (13).More than $90 \%$ childhood HIV infections are due to MTCT thus 600,000 children are newly infected by HIV worldwide annually $(14,15)$. For instance, In Ethiopia, HIV prevalence among cohabiting individuals is notably high in urban areas (10.9\%); of whom about (72\%) of the cohabiting couples are discount. However, In Urban areas, $5.6 \%$ of HIV negative married men are living with infected wives and $2.2 \%$ of married HIV negative women are living with infected husbands (16). The current adult prevalence of HIV in India is $0.34 \%$.HIV infected persons should have adequate knowledge about the modes of transmission of infection. This is essential for reducing the risk of secondary infection, preventing co infection from other viruses such as hepatitis B and C viruses (17). There are an estimated 34 million people around the world who are living with $\mathrm{HIV}$, including millions who have developed AIDS. In 2010, around 390,000 children under the age of 15 became infected with HIV; mainly through mother -to-child transmission about $90 \%$ of children living with HIV reside in Sub-Saharan Africa where, in the context of a high child mortality rate, AIDS accounts for $80 \%$ of all under -five deaths in the region (18). In addition, $90 \%$ of the estimated 1,000 children uninfected daily with HIV live in Sub-Saharan Africa. Vertical transmission accounts for approximately $95 \%$ of infectious in Children $(19,20)$. The implementations of national PMTCT of HIV programs have been studied in several sub-Saharan countries. Women's program attendance has been studied in Kenya, Ethiopia, Zimbabwe, Malawi, and South Africa $(21,22)$. In 2008, 57 countries documented the number of male partners of pregnant women attending antenatal care who received on HIV test. The proportion of pregnant women attending antenatal care whose male partners were tested for HIV was $5 \%$ in 2008 versus $2 \%$ in 2007(23). In addition, men can also play a crucial role in supporting HIV positive pregnant women, by assisting them 
to get to clinics or Hospitals where chances of safe delivery are higher, and to choose a safe infant feeding method (24).

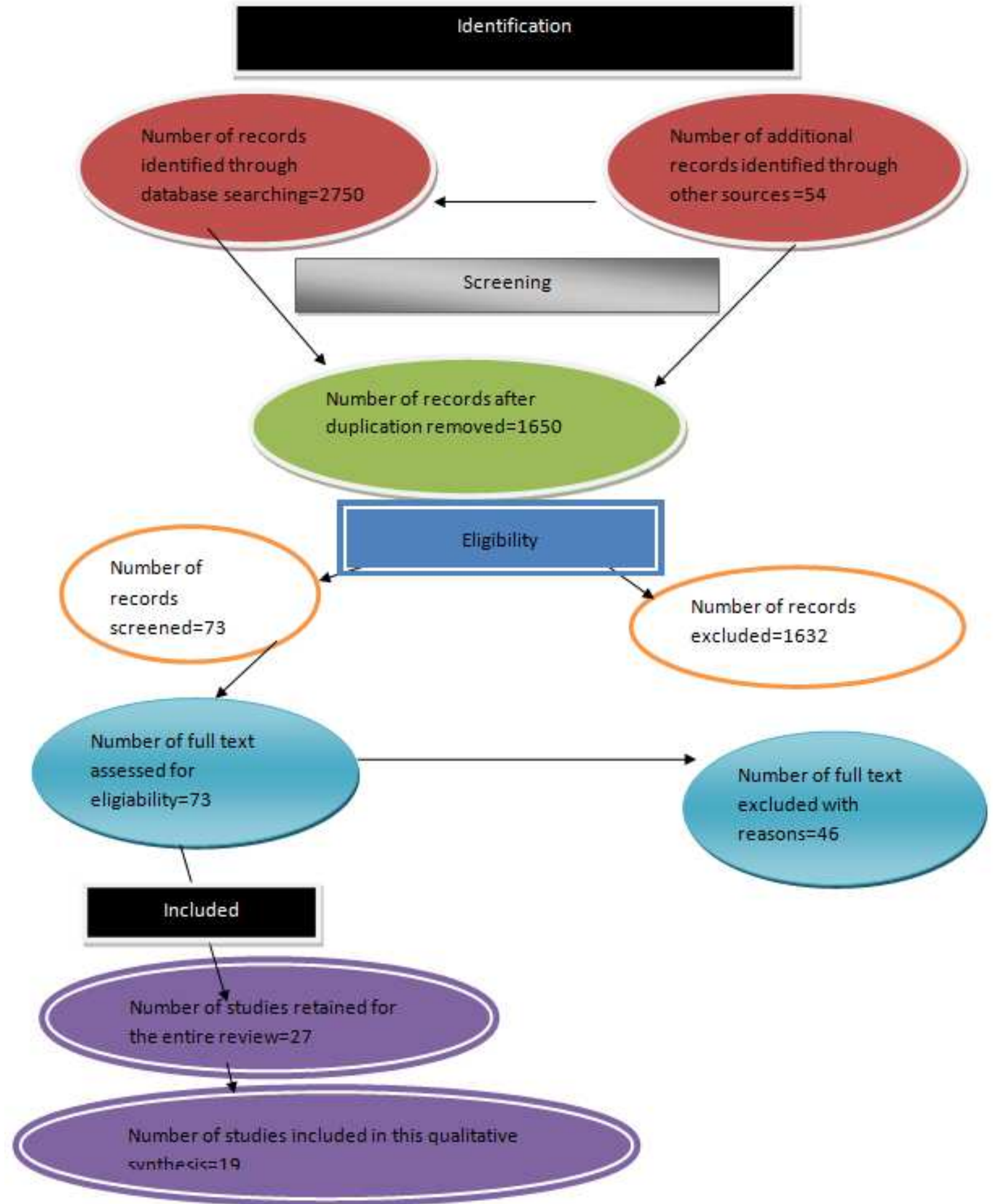

Fig. 1. PRISMA diagram for the flow of information through different phases of the review.

\subsection{Level of Male Involvement in PMTCT, Importance, Utilization and Adherence of PMTCT Services}

Male partner participation is a crucial component in the optimization of PMTCT services. For this reason, ANC/PMTCT is the only opportunity to capture the transmission of HIV during pregnancy, labor, and breast feeding. Male involvement in the PMTCT of HIV services is essential in a patriarchial society where men are key decision makers as in most African countries $(25,26)$. Male participation has been found to be an important factors for the mothers, in the overall use of PMTCT of HIV programs 
$(27,28)$.Important strides have been made in recent years in the PMTCT of the HIV. Yet, despite these advances, approximately $15 \%$ of all new cases of HIV infection have been diagnosed in children in developing countries (29). The importance of male involvement in PMTCT is accepted globally, but few programs incorporate male involvement even in family-centered models of care, are documented as growing (30). Furthermore, the importance of male involvement in the prevention of mother-to-child transmission programme is incremental to maintain family health and adherence to human immunodeficiency virus treatment and prevention has been recognized as a priority focus area to be strengthened in prevention of mother-to child transmission but, it remains a challenge in most low-andmiddle income countries including Ethiopia (31). Most males do not participate in PMTCT programs because they do not realize their importance due to inadequate knowledge about the programs, while in the community which pose negative perceptions towards the programs(32).Providing suitable medical information to men has several important consequences related to PMTCT interventions: Wellinformed men will be more likely to participate positively in the decision making for the well-being of the couple, women with supportive partners will be more motivated to undergo HIV testing, to return for the HIV test result and to discuss the HIV result to their partner , and well-informed couple may be more likely to adopt a low risk behavior and increase mutual support, regardless of the test result $(33,34)$. Utilization and Adherence of PMTCT services by the pregnant women is influenced by both factors related to the health system such as accessibility of voluntary counseling and testing (VCT) services and individual factors such as fear of disclosure of HIV results and lack of male partner support(35). According to EDHS 2011 only $34 \%$ of mothers had ANC follow up in Ethiopia, thus having a negative contribution on under-utilization of PMTCT services .Better knowledge of, good attitude towards and practicing PMTCT is highly effective intervention and has an enormous potential to improve both maternal and child health(36).

\subsection{Knowledge about PMTCT and Reason that Rationalize Male Partner Involvement in ANC/PMTCT}

The main role of men can play in the PMTCT of HIV is cardinal in changing the course of the outbreak of the diseases. In addition, men take part in the PMTCT of HIV, their knowledge of HIV increases; they become supportive to improve $(37,38,39)$. HIV/AIDS education aimed at increasing knowledge and PMTCT awareness among clients/partners within the community is still low. Stigmatization is singled out as a matter to be addressed (40, 41). Better knowledge of, good attitude towards and practicing prevention of mother-to-child transmission is highly effective intervention and has an enormous potential to improve both maternal and child health $(40,41)$. The narrow focus of PMTCT to date which represents a lost opportunity to effectively cobalt the vertical transmission of HIV to children-a largely preventable infection given current scientific knowledge (42). There is an over growing discordant rate among couples .For instance, the study finding which was conducted in Ethiopia showed that effective PMTCT interventions, male partners should be involved in their wives ANC/PMTCT. In addition. Low male partner's involvement in PMTCT, stigma and discrimination, and fear of testing positive for HIV as reasons for refusing to test for HIV (43).

\subsection{Determinants of Male Involvement and Association Between Male Partner Involvement in PMTCT and the Uptake of PMTCT Intervention}

Involvement of male partners may increase adherence to PMTCT and its program outcomes . Therefore, male partners participation was associated with positive outcomes: greater use of ARV therapy, higher acceptance of post-test counseling among pregnant women, increased spousal communication about HIV and safe sex (44).Male participation in child -bearing decisions is crucial and also has a positive impact on the acceptability of PMTCT interventions (44). Even though there is positive impact on the uptake and adherence to PMTCT regimens especially when men accompany their partners, evidence indicates that only a few men accompany their female partners for antenatal care and participate in PMTCT programmes with rate of $3.2 \%$ in Malawi (45). Male partner involvement may improve uptake of prevention of mother-to-child HIV-1 transmission (PMTCT) Interventions and HIV-free child survival (46). Male partner involvement increases the uptake of some PMTCT interventions by HIV positive women.Multistrategic, culturally tailored public health care models are needed to increase the rate of male partner involvement in the program (47).

\subsection{Husbands Accompany Their Wives to the ANC/PMTCT Clinic, Challenges Facing the PMTCT Programs}

Detection of maternal infection in early pregnancy through provider initiated HIV testing and counseling (PITC) is not enough to mitigate mother to child transmission of HIV but only few husbands accompany their wives to the ANC/PMTCT clinic $(48,49)$. Efforts to involvement men in ANC services, where PMTCT takes place ,have only resulted in a few husbands being involved in PMTCT services have been critized for only focusing on females and sidelining males who are the primamary support unit to the women(50). Since husbands play a pivotal role in decision -making within the home, and are often the main bread winners, establishing their buy-in and support for PMTCT activities and interventions is critical $(51,52)$. Husband's role if a likely determinant for the successful implementation of PMTCT guidelines/standards in sub-Saharan Africa (53). The challenges facing the PMTCT programmes in sub Saharan Africa are numerous including large proportions of home deliveries, fear of the cultural implication of a positive HIV test result, such as the lack of male partners support and even violence (54). Barriers of male involvement in PMTCT 
services are categorized into: Health systems, community level and personal and family factors. Health system barriers include the clinic set up, using women to convey messages to men, services costs and distance to the clinic (55).

\subsection{Programmatic Factor or Male Friendly of the Health Facility, Husbands Accompany Their Wives to the ANC/PMTCT Clinic and Strategies of PMTCT}

With Kwazulu-natal being the province worst affected in South Africa by the disease burden of HIV and AIDS 38.7\% of pregnant women attending ANC tested positive for HIV in 2008 according to the south Africa National Department of health -the lack of male partner involvement has been seen the contributing factors to a poor programme adherence by women who are initiated into the prevention of mother-tochild- transmission (PMTCT) programme in South Africa (56). Action shown to facilitate male in PMTCT involvement were either health system actions or factors directly tied to the individuals. Inviting men to the hospital for voluntary counseling and HIV testing and offering of PMTCT services to men at sites other than antenatal care were key health system facilitators (57). PMTCT strategies introduced in 2001, have become integrated into all maternal and child health services (58). The strategy on the need for government and health workers to create a conductive environment in Antenatal clinics for male partners' participation was found to be effective in Burkina Faso and Malawi (59). Male participation is a crucial component in the optimization of maternal and child health (MCH) services .This is especially so where prevention strategies to decrease mother-to-child transmission (MTCT) of Human Immunodeficiency Virus (HIV) are sought $(60,61)$.

\section{Conclusion}

The prevalence of PMTCT, Associated between male partner's involvement, and the uptake of PMTCT intervention, Determinants of male involvement in PMTCT, Knowledge about PMTCT, Utilization and adherence of PMTCT services, PMTCT strategies, Reason that rationalize male partner involvement in PMTCT/ANC, challegnes facing the PMTCT programs, Husbands accompany their wives to the ANC/PMTCT clinic, Level of male involvement in PMTCT, Implementations of PMTCT, programmatic factors(male friendly of the health facilities), and Importance of PMTCT were identified, each having its own attributes and uses. The current literature, research and reviewed articles which were developed through an evaluation of this literature reviewed articles and the assessment of a limited number of research studies that focused on male partner's participation in PMTCT of HIV and associated factors in PMTCT services at different health settings.

\subsection{Implication of PMTCT Services}

It is proposed that male partner's participation in PMTCT of HIV improves as the PMTCT services gains HIV testing experience in their facilities with couples, male partner's gain a sense of saliency in relation to PMTCT services. Male partners may use PMTCT services independently, and concurrently to solve weak and longtime PMTCT services at different health Institutes.

\subsection{Strength and Weakness}

The strengths of the article is that the review is focus on the situation of public health in developing country, such as Sub-Saharan Africa Countries, and the findings generally make a contribution to the field ,particularly providing the information of the prevention of mother-to-child transmission of HIV and the related impact factors.

The weakness of this paper is the outlines of the review is a little bit confusing and lack of logical relationships. Overall this is a meaningful review but needed to be recognized following a more reasonable orders.

\section{Acknowledgement}

It is hereby acknowledged colleagues and all those who associated in conducting the study or critiquing the manuscript.

\section{Author's Contribution}

Development of the original idea and protocol, data abstraction and analyses, writing the manuscript: Addis Adera Gebru, Mesfin Wudu Kassaw, and YonasYimama Ayene: and Development of the protocol, over all Guide data abstraction, preparing the manuscript: Markos Kidane Assefa, Zemenu Mengistie Semene, Ambachew Woreta Hailu

\section{References}

[1] Auvinene, J., Kylma, J., Valimaki, M., Buleupe, M., Suominen, T (2014). Luba-Kasai men and the prevention of mother to child transmission (PMTCT) of HIV program in Lusaka. Health promotion International Advance Access Published . Doi: 10.1093/heapro/data 088.

[2] WHO (2010). Towards universal access: Scaling up HIV services for women and children .Progress Report. http:// www.who.int/hiv/topics/mtct/en.last accessed 04, April, 2014.

[3] Alemayehu,M.,Etana,B.,Fisseha,G.,Haileselassie,K.,Yebyo,H. ,Berhe,Y.,Ashebir,F(2014).The role of male partner involvement on mother's adherence of PMTCT care support, Tigray, Northern Ethiopia. Fam Med Sci. Res:3(4):1-7. http://dx.doi.org/10.4172/2327-4972.1000137.

[4] Pelzer, K., Jones, D., Weiss, SM., Shikwane, E (2011). Promoting male involvement to improve PMTCT uptake and reduce antenatal HIV infection: a cluster randomized controlled trial protocol. BMC Public Health, 778, DOI: 10.1186/1471-2458-11

778.http://www.biomedcentral.com/content/pdf/1471-245811-778.pdf. 
[5] Awungafac, G., Njukens, PA., Ndasi, JA., Mbuagbaw, LT (2015). Prevention of mother-to-child transmission of the Human Immunodeficiency virus: Investigating the uptake and utilization of maternal and child health services in Tiko health District, Cameroon. Pan African MedicalJournal.20:20.Doi:10 .11604/pamj.2015.20.20.5137.http://www.panafrican-medjournal.com/context/article/20/20/full.

[6] World Health Organization. Education of maternal mortalityA joint WHO/UNFPA/UNICEF/World Bank Statement. Geneva Switzerland, 2014.

[7] WHO/UNICEF/IATT (2007). Guidance on the global scale up of the prevention of mother-to-child-transmission of HIV .Top wards universal access for women, infants and young children and eliminating HIV and AIDS from among children.

[8] Haile, F., Brahn, Y (2014). Male partner involvements in PMTCT: a cross sectional study, Mekelle, Northern Ethiopia. BMC pregnancy and childbirth. 14:65:16.http://www. BioMed icenral.com/1471-2393/14/65.

[9] Byamugisha, R., Tumwine, JK., Semiyaga, N.,T Ylleskal, T (2010). Determinants of male involvement in the prevention of mother -to-child transmission of HIV programme in Eastern Uganda: a cross sectional Survey. Reproductive Health, 7:12:1-9. http://www. Reproductive-health -Journal. com/Content/7/1/12.

[10] Nyondo, AL., Chimwaza, AF., Muula, A.S (2014). Stakeholders' perceptions on factors influencing male involvement in prevention of mother to child transmission of HIV services in Blantyre, Malawi. BMC Public Health: 14:691:1-15. Http://www.biomedcentral.com/1471$2458 / 14 / 691$.

[11] Perez(2010). Factors influencing male involvement in prevention of mother to child transmission of HIV program among Luzira's prison custodial Staffs, Kampala, Uganda. http://hdl.handle.net/10570/1303

[12] Ministere De La Sante Publique (2015). Ver elimination De la Transmission DU VIH de la Mereal'enfant A l' horizon 2015.Rapport de progress no5.2010, Cameroon.

[13] Nkuoh, GN., Meyer, DJ., Tih, PM., Nkfusai, J(2010). Barriers to men's participation in antenatal PMTCT care in Cameroon, Africa .J Midwifery Women's Health .55(4): 363-9.

[14] FMOH/FHAPCO (2007). Guidelines for implementation of the antiretroviral therapy program in Ethiopia. Addis Ababa, Ethiopia: FMOH; 2007.

[15] FMOH/FHAPCO (2007).Training manual draft for the prevention of MTCT of HIV in Ethiopia. Addis Ababba,Ethiopia.FMOH;2007.http://www.ilo.org/Wcmsps/gro ups/public/ed-protect/-protrav/-ilo-addis/docuent5s/legal documents/wcms-125385.pdf.access in April, 2015.

[16] HAPCO/GAMET: HIV/AIDS in Ethiopia an epidemiological synthesis, the global HIV/AIDS program. Addis Ababa Ethiopia: 2005 Available at http://siteresources worldbank.org/INTHIV/AIDS/Resources/375798-

$1103037 / 53392 /$ Ethiopia synthesis Final. pdf. Accessed in January, 2011.

[17] Mohalsksmy, T., Premarajan, KC., Alamide, A(2011). Correlates of human immunodeficiency virus (HIV) related knowledge among HIV infected people. Indian Journal of Dermatology, Venereology and Leprology. 77 (1):37.
[18] UNAIDS (2010). UNAIDS report on the global AIDS epidemic .Global report, Switzerland, Geneva: Joint United National Program on HIV /AIDS.

[19] Lemens, CL (2010). Male partner involvement in PMTCT reduces HIV transmission risk .Http://www.aidsmap.com/male - partner-involvement-in-PMTC-reduces-HIV-transmission risk/Page/1581480/.

[20] Aluiso et al (2011). Male antenatal attendance and HIV testing are associated with decreased infant HIV infection and increased HIV-free survival. J Aquir Immune Defic Syndr.56 (1): 76-82.

[21] Kalembo,F., Zgambo, M., Mulaga, A., Yukai, D., Ahmed, NI ( 2013). Association between male partner involvement and the uptake of prevention of mother-to-child transmission of HIV (PMTCT) interventions in Mwanza District, Malawi. A Retrospective Cohort study. PLOS one; 8(6):e66517: doi: 10.1371/Journal-pone0066517.

[22] Osoti, A., John Stewart, G., Richardson, B.,James, K., Konuthia, J., Krakowiak-Redd, D., Farquhar,C(2012). Home visits during antenatal care enhance male partner HIV-1 counseling and testing during pregnancy in Kenya: A randomized control trial. TUACO103-oral abstract session.

[23] Kalembo, FW., Zgambo, M., Mulaga, AN., Yukai, D.,Ahmed, NI (2013). Association between male partner involvement and the uptake of prevention of mother to child transmission of HIV(PMTCT) interventions in Mwanza District ,Malawi: A retrospective Cohort study.PLOS/ONE.8(6):e66517.http://ww w.plosone.org

[24] AIDS in Ethiopia. National Factsheet main Indicators .Addis Ababa, Ethiopia, 2010.

[25] Motongo, LH (2014).Exploring determinants of male involvement in PMTCT programs in Chibombo district of central province of Zambia. Asian Journal of Multidisciplinary studies. 2 (12):1-5.

[26] Aarnio, P., Plsson, P., Chimbri, A., Kulmala, T (2009).Male involvement in antenatal HIV counseling and testing: Exploring men's perceptions in rural Malawi. AIDS Care, 21(12):1537-1546.

[27] Higgins, JA., Dworkin, SL (2010). Rethinking Gender, Heterosexual men and women's Vulnerability to HIV/AIDS. American Journal of public health, 2010.

[28] Akarro, RRJ., Deonisia, M., Sichona, FJ (2011). An evaluation of male involvement on the programme for PMTCT of HIV/AIDS: A case study of Ilala Municipality in Dares Salaam, Tanzania. Art and social Sciences Journal: 1.Accession No_90253867.

[29] Abajobor, A A., Zeleke, AB (2013) .Knowledge, Attitude, practice and factors associated with prevention of mother-tochild transmission of HIV/AIDS among pregnant mothers attending Antenatal clinic in Hawassa Referral Hospital, South Ethiopia. J AIDS Clin Res. 4(6):1-7.http://dx.doi.org/10.4172/ 2155-6113.1000215.

[30] Maman, S., Mbwamboo, J., Hogan, MN., Kilongo, GP., Sweat, $\mathrm{M}(2010)$. Women's barriers to HIV-1 testing and disclosure: Challenges for HIV-1 voluntary Counseling and testing AIDS Care 13:595-603.

[31] Central Stastical Agency Addis Ababa Ethiopia (2012). Ethiopia Demographic and Health Survey 2011. ICF International Caverton, Maryland, USA. 
[32] Kassenga, F., Peter, B., Maria, E., Hurtog, AK (2009). The implication of policy changes on the uptake of a PMTCT programme in rural Malawi: First three years of experience. Global Health Action. Jan 23:2.

[33] Njunga, J., Blystad, A (2010). The divorce program: Gendered experiences of HIV positive mothers enrolled in PMTCT program-the case of rural Malawi. International Breast Feeding Journal 5(4): Doi: 10.1186/1746-4358-5-14.

[34] Ditekemena, J. Koole, O., Engmann, C., Matendo, R., Tshefu, A., Ryder, R., Colebuders, R. (2012). Determinants of male involvement in maternal and child health services in SubSaharan Africa: a review -reproductive Health. 9(32):1-8. $\mathrm{http}: / /$ www.reproductive health -journal .com/content/9/1/32.

[35] Robert, B., James, T., Nulu, S., Thorkild, T (2010). Determinants of male involvement in the prevention of mother -to-child transmission of HIV programme in Eastern Uganda. BMC Reprod Health. 7(11): 1-6. Doi. 10.1186/1742-47-55-7-12.

[36] Kalembo, F. W., Yukai, D., Zgambo, M., Jun, Q (2012). Male partner involvement in prevention mother-to-child transmission of HIV in sub-Saharan Africa: success challenges and way-forward. OJPM. 2(1):35-42.

[37] Kasenga, F., Hurtiga, K., Emmelin, M (2007). Home deliveries: Implications for adherence to nevirapine in a PMTCT programme in Rural Malawi. AIDS Care19 (5): 64652.

[38] Tweheyo, R., Konde-Lule, J., Tumwesigye, N.M., Sekandi, JN (2010). Male partner attendance of skilled antenatal care in peri urban Gulu District, Northern Uganda. BMC Pregnanacy Childbirth. 10(53)::1-8.

[39] UNAIDS/WHO (2011). Global AIDS update. USAIDS; 2009. Available at http://data.Usaids.org/pub/press realse /2009/20091124-PR-Epiupdate-en.pdf. Accessed in April, 2015.

[40] Koo, K., Makin, J., Fosyth, B (2013).Where are the men? Targeting male partners in preventing mother-to-child transmission AIDS Care. 25 (1):43-48.

[41] Reece, M., Hollub, A., Nangami, M., Lane, K (2010). Assessing male spousal engagement with prevention of mother-to-child transmission (PMTCT) program in women in Western Kenya. AIDS Care: 22(6):743-750.

[42] Byamagisha,R., Astrom, AN.,Ndeezi,G.,Karamagi,CA.,Tylles kar,T.,Tumwine,JK(2011). Male partner antenatal attendance and HIV testing in eastern Uganda: a randomized facility based intervention trial. J Int AIDS Soc. 14(1):43.

[43] Ditekema,J.,Matendo,R.,Koole,O.,Colebunders,R.,Kashamuka ,M.,Tshefu,A.,Kilese,N.,Nanele,D.,Ryder,R(2011). Male partner Voluntary counseling and testing associated with the antenatal services in Kinshasha, Democratic Republic of Congo: a randomized controlled trial. Int J STD AIDS. 29 (3): 165-170.

[44] World Health Organization (2010). Antiretroviral drugs for treating pregnant women and preventing HIV infection $s$ in infants. Recommendations for a public health approach. Geneva 2010. http://www.who.int/hiv/en

[45] Kura, S., Vince, J., Crouch-Chivers, P (2013). Male involvement in sexual and reproductive health in the Mend district, Southern Highlands province of Papua New Guinea: a descriptive study. Repod Health Matters. 10(1):46.
[46] Kassenga, F (2012). Perceptions about barriers and promoting factors among services providers and community members on PMTCT services. Electronic article accessed from: WWW.intechnoprn.com/books/hiv-testing/perceptions-about -barriers-and-promoting-factors-among-service-providers-and community-members-on pm, last accessed on 22, April, 2015

[47] Bolu,O.,Anand,A.,Swartzendruber,A.,Hladik,W.,Marum,LH., Shcikh,AA.,et al(2007). Utility of antenatal HIV surveillance data to evaluate prevention of mother -to-child transmission programs in resource limited settings. American Journal of Obstetrics and Gynecology, 197, 517-525.

[48] Chinkonde, JR., Sundby, J., Martinson, F (2009). The prevention of Mother-to-child HIV transmission programme in Lilongue, Malawi: Why do so many women drop out. Reproductive health matters. 17. 143-151.

[49] WHO, UNICEF (2007).New Guidance on global scale up of the prevention of mother to child transmission of HIV: towards universal access for women, infants and young children and eliminating HIV/AIDS among children. Switzerland: World Health Organization HIV/AIDS Department.

[50] Malawi Ministry of Health (2008).PMTCT of HIV and pediatric HIV care gudelines. $2^{\text {nd }}$ edition, Ministry of Health Lilogue. Available: http:// www.aidstar-one.com/sites /default/files/PMTCT \%20 Guidelines 5\% 20 Malawi\%20 2008 pdf. Accessed, 2015 April, 22.

[51] Jeewa, Z (2014). Male partner involvement highlighted in KZN study on pregnant women.2919):12.http://ndaboline.ukzn.ac.za/ukzndabastory/Ndabaonlinevol2-Issue-19-college-of hs/male $\% 20$ partner $\% 20$ involvement $\% 20$ highlighted $\% 20$ in $\% 20$ KZN\% 20 study $\%$ 20 on $20 \%$ pregnant $\% 20$ Women.

[52] Morfaw, F., Mbuagbaw, L., Thabane, L., Rodrigues, C., Wunderlich, AP., Nana, P., Kunda, J(2013). Male involvement in prevention programs of mother-to-child transmission of HIV: a systematic review to identify barriers and facilitators. Systematic Review Journal. com/content/2/1/5. doi. 10.1186/2046-4053-2-5.

[53] Musuya,SE.,Mbizro,EM.,Hussain,A.,Uriyo,J.,Sam,NE.,StrayPedersen,B(2008). Low male partner participation in antenatal HIV counseling and testing in Northern Tanzania: Implications for preventive programs. AIDS Care 20: 700-709.

[54] Kasenga, F., Hurtig, AK., Emmelin, M (2010). HIV positive women's experiences of a PMTCT programme in rural Malawi. Midwifery, 26:26-37.

[55] AIDS epidemic update (2009). December 2009 Geneva: Joint United Nations Program on HIV/AIDS and World Health Organization

[56] Auvinene, J. MKylma, J., Suominen, J (2013). Male involvement and prevention of mother to child transmission of HIV in Sub -Saharan Africa: An integrative Review. Current HIV Research ,11(2): 169-177.http://oucsupport.net/maleinvolvement-and-prevention-ofmother-to-child-transmission of hiv-in sub-Saharan-Africa-an-integrative-review/

[57] Abuhay, Y., Abebe, L., Fetntahun, N (2014). Male involvement in prevention of mother -to-child transmission of HIV and associated factors among males in Addis Avbabba, Ethiopia. Science Publication Group American Journal Of health Research. 296): 338-343. doi. 10.11648/j.ajhr.20140206.13. 
[58] Boniphace, Y (2009). Willingness and participation towards prevention of mother to child transmission among males of reproductive age. A study from Kilimanjaro, Tanzania. Official publication of the Tanzania MNedical Students' Association. MDSMUHAS-2008/2009.

[59] Koo, K., Makin, J., Forsyth B., et al(2010). Where are the men? Understanding male involvement in the prevention of mother -to-child transmission. Vieenna: International AIDS Conference. Abstract No MOPE0255.

[60] Duff, P., Kipp, W., Wild, TC., Rubaale, T., Okech-ojony, J
(2011). Barriers to male partner involvement in PMTCT programme and HIV testing: a need for male partner friendly services, $6^{\text {th }}$ IAS conference on HIV pathogenesis and Treatment, and prevention. ROME .Abstract No CDC031.

[61] Godana, W., Atta, A(2013).Prevalence of HIV/AIDS and its Associated Factors among Prevention of Mother-to-Child Transmission (PMTCT) Service Users in Jinka Town Health Institutions, South Omo Zone, South Ethiopia. Science Journa $l$ of Public Health. 1(3): 125-130. Doi: 10.11648/j.sjph.20130103.13. 